\title{
On the increase of sweetness under zero gravity
}

\author{
ERNEST DZENDOLET \\ University of Massachusetts at Amherst, Amherst, Massachusetts
}

\begin{abstract}
The reported increase in the sweetness of stimuli when tasted in zero gravity is analyzed with respect to the effect of gravity on the free-energy change of a chemical reaction. In the analysis, I question the assumption that the initial gustatory step for sweet is a stimulus-receptor site association. An earlier alternate hypothesis-a dissociation process-is reintroduced, and predictions are made for the responses of other gustatory qualities under zero gravity. The possibility is noted that this variation in the free-energy change of a reaction may have an effect on other biochemical processes.
\end{abstract}

In an interview with the New York Times (Reinhold, 1985), Robert L. Stewart, mission specialist on the February 1984 space-shuttle flight, noted that sweetened drinks, when tasted on board the shuttle under zero-gravity conditions, appeared to evoke a much sweeter sensation than when they were tasted on Earth. In this paper, I suggest a basis for Stewart's observation; namely, I point out that the free-energy change of a chemical reaction depends upon the strength of the gravitational field in which the reaction takes place. In addition, I present an argument against the generally accepted mechanism for the initiation of the sweet quality.

\section{HYPOTHESES ON THE INITIAL GUSTATORY STEP}

The general mechanism for the initiation of any taste sensation is considered to be the temporary binding of a stimulus molecule with a particular site on the gustatory receptor membrane. This view was first advanced by Renqvist (1919), who considered the attachment to the membrane to be one of adsorption. Beidler (1954), however, assumed that there was a chemical reaction between the stimulus and the receptor site and that this reaction could be quantified by applying the law of mass action to this process. Using electrophysiological data obtained from the rat, Beidler was able to calculate the equilibrium constant, and the related change in free energy, for the presumed reaction. Although the change in free energy was negative, indicating that the assumed reaction would take place spontaneously, its value was low. Because of this, Beidler also viewed the taste process as one of adsorption rather than of a chemical reaction. In addition, Beidler assumed that the intensity of the sensation was a direct function of the number of receptor sites to which the stimulus molecules were attached.

With regard to the quality of sweet, Shallenberger and Acree (1967) also assumed that a stimulus-receptor product has to be formed. Specifically, Shallenberger and

The author's mailing address is: Department of Psychology, Tobin Hall, University of Massachusetts, Amherst, MA 01003.
Acree assumed that the stimulus has to possess a paired proton-donor, proton-acceptor structure that would attach itself to a similar but reversed structure on the gustatory membrane.

\section{THE INFLUENCE OF GRAVITY ON THE FREE-ENERGY CHANGE}

The influence of gravity on a chemical reaction can be seen in the results of an experiment reported by Tolman (1911). In that experiment, an electric cell was centrifuged. Its voltage increased considerably over its noncentrifuged or normal-gravity value. The voltage of a cell is related to the change in the free energy of the reaction involved as follows:

$$
-\Delta \mathrm{G}=n F e,
$$

in which $\Delta \mathrm{G}$ is the change in the free energy, $n$ is the number of equivalents involved in the reaction, $F$ is the value of the Faraday, and $e$ is the voltage. The values of the parameters $n$ and $F$ are not important for the purposes of the current argument. What is important is that an increase in the voltage reflects an increase in the negative free-energy change. Furthermore, the change in free energy is related to the equilibrium constant of a reaction, as follows:

$$
-\Delta \mathrm{G}=R T \ln \mathrm{K},
$$

in which $R$ is the gas constant, $T$ is the termperature, and $K$ is the equilibrium constant. Again, only $\Delta G$ and $K$ are important. $K$, in its turn, is defined as the ratio of the result of multiplying together the concentrations of the products of a reaction, to the same treatment of the concentrations of the reactants of that reaction. Depending on the specific reaction, either the numerator or the denominator of the ratio could be the concentration of a single chemical species. Furthermore, if $K$ is positive and greater than unity, such a value signifies that the reaction proceeds spontaneously in the direction of the product(s), and that the associated change in free energy is negative. The above equation can also be used from the point of view of the free energy. A larger value of the negative free energy 
would also indicate a greater movement of the reaction in the direction of the product(s).

The results of Tolman's (1911) experiment can be interpreted as showing that the value for the negative free energy of a reaction increases directly with an increase in the strength of the gravitational field. This increase also means that the reaction goes further in the direction of the products of that reaction (i.e., the value of $K$ increases). This being the case, it can now be argued that a decrease in the value of the gravitational field below its normal value will give rise to the opposite result, that is, a decrease in the value of the negative free-energy change and a decrease in the products of a reaction.

\section{APPLICATION TO THE INITIAL GUSTATORY STEP}

The reduction, under zero gravity, in the value of the negative free-energy change of the presumed initial gustatory step would lead to a reduction in the number of stimulus-receptor site complexes. This smaller number should, according to Beidler's (1954) view, lead to a decrease in the intensity of the sensation of sweet. On the contrary, the space-shuttle report is of an increase in the intensity of that sensation. Therefore, the presumed initial step, at least for sweet, does not appear to be correct.

\section{AN ALTERNATE HYPOTHESIS FOR THE INITIAL "SWEET" STEP}

The following proposal was advanced by Dzendolet in two articles. In the first one, Dzendolet (1967) reviewed Beidler's (1954) application of the law of mass action and also derived a relation between the concentration of the stimulus and the electrophysiological response. This relation, however, was more complicated than Beidler's, because it was exact; that is, it was without the approximation that Beidler had assumed in his derivation. In reanalyzing the same physiological data that Beidler had used, Dzendolet concluded that the reaction at low salt concentrations (i.e., those which are reported by humans as being sweet) was not the formation of a stimulusreceptor complex, but rather the dissociation of a complex which was already present. Calculation of the change in free energy for this situation yielded a value that was close to the one found by Beidler. In his second article, Dzendolet (1968) proposed that a sweet-evoking stimulus needs to be only a proton-acceptor, and not the proton donor-acceptor pair suggested by Shallenberger and Acree (1967). Furthermore, the dissociation which Dzendolet (1967) proposed could now be seen to be the removal of a proton from its attachment to a receptor site. The source of these protons is assumed to be the various species of carbonates and phosphates that are normally present in the saliva. The equilibrium state of a chemical reaction has been interpreted for over 100 years as the result of two opposing rates of reaction: a forward one and a reverse. In Dzendolet's model, the forward reaction is a dissociation (i.e., the removal or release of the protons from their membrane sites). Under zero gravity, the extent of this forward reaction would be reduced or the reverse reaction increased, leading to a greater concentration of the proton-site complex (i.e., more receptor sites would be occupied by protons). Therefore, more protons would be available to be pulled off by the stimulus. With more being pulled off, the stimulus would taste sweeter than it would under normal conditions. This situation is similar to rinsing the mouth first with an acid solution, thus loading up the "sweet" sites, and then rinsing the mouth with water. The rinse water tastes sweet, simply because it removes protons from their receptor sites as a consequence of the difference in the concentrations of the protons in the rinse water and on the gustatory membrane.

\section{POSSIBLE TESTS OF THE ALTERNATE HYPOTHESIS}

The current data on the increase in sweetness is essentially anecdotal, but that increase must have been significant for it to have been so easily noticeable. Nonetheless, it would be important to have further tests carried out on future shuttle flights in order to confirm the observation. In addition, it would also be important to test the other taste qualities for possible changes, to see if these changes occur in the same or in the opposite direction as that for sweet. Alternatively, such tests could be carried out on the surface of Earth in a centrifuge.

I advance the following hypothesis. If, as suggested by von Békésy (1964), there are two gustatory senses, sweetbitter and sour-salty, it seems reasonable to assume that these two senses would operate according to similar general principles. If the sweet quality results from the removal of a compound from a receptor site, then a similar process should take place in the sour-salty system. I suggest that this process is the one which initiates the salty quality. This means that the sour and the bitter qualities result from an association of their stimuli with their receptor sites, whereas the sweet and salty qualities result from a dissociation. An observation which is consistent with this view concerning the salty quality is that a solution of urea evokes a bitter taste, but that a subsequent water rinse evokes a salty taste (McBurney \& Shick, 1971). The similarity between this observation and the one mentioned earlier concerning an acid stimulus and a following water rinse is obvious.

If the above hypothesis is correct, the prediction for zero gravity is clear. The intensities of the sweet and salty qualities should increase, whereas the intensities for the bitter and sour should decrease.

The effect of zero gravity on the equilibrium constant of a reaction should not be assumed to be limited to the special case of the gustatory system. All chemical reactions would be affected, to either a greater or a lesser 
degree; but the most important effect would be on the various biochemical cycles taking place within living organisms. Changes in the functioning of various body tissues exposed to zero gravity have been reported at meetings concerned with gravitational or space physiology (e.g., Commission on Gravitational Physiology, Niagara Falls, New York, October 18, 1985). It seems reasonable to suppose that the sources for such changes would be related to the differences between the free-energy changes of reactions taking place on Earth, and those in space.

\section{REFERENCES}

BemLER, L. M., (1954). A theory of taste stimulation. Journal of General Physiology, 38, 133-139.

Dzendolet, E. (1967). Beidler's taste equation: A correction. Perception \& Psychophysics, 2, 519-520.
Dzendolet, E. A. (1968). A structure common to sweet-evoking compounds. Perception \& Psychophysics, 3, 65-68.

MCBURNEY, D. H., \& SHICK, T. R. (1971). Taste and water taste of twenty-six compounds for man. Perception \& Psychophysics, 10 , 249-252.

ReINHOLD, R. (1985, January 16). Dining a la carte in the space shuttle. New York Times, p. C17.

RENQVIST, Y. (1919). Über den Geschmack. Skandinavisches Archiv der Physiologie, 38, 97-201.

Shallenberger, R. S., \& ACReE, T. E. (1967). Molecular theory of sweet taste. Nature, 216, 480-482.

Tolman, R. C. (1911). The electromotive force produced in solutions by centrifugal action. Journal of the American Chemical Society, 33, 121-147.

voN BÉKÉsY, G. (1964). Duplexity theory of taste. Science, 145, 834-835.

(Manuscript received for publication March 24, 1986.) 